\title{
Simple cyclic covers of the plane and the Seshadri constants of some general hypersurfaces in weighted projective space
}

\author{
Alex Küronya $^{1} \cdot$ Sönke Rollenske ${ }^{2}$ (D) \\ Received: 30 November 2020 / Accepted: 30 April 2021 / Published online: 8 May 2021 \\ (c) The Author(s) 2021
}

\section{Abstract}

Let $X \subset \mathbb{P}(1,1,1, m)$ be a general hypersurface of degree $m d$ for some for $d \geq 2$ and $m \geq 3$. We prove that the Seshadri constant $\varepsilon\left(\mathcal{O}_{X}(1), x\right)$ at a general point $x \in X$ lies in the interval $\left[\sqrt{d}-\frac{d}{m}, \sqrt{d}\right]$ and thus approaches the possibly irrational number $\sqrt{d}$ as $m$ grows. The main step is a detailed study of the case where $X$ is a simple cyclic cover of the plane.

Keywords Seshadri constant $\cdot$ Hypersurface $\cdot$ Multiple plane

Mathematics Subject Classification $14 \mathrm{C} 20 \cdot 14 \mathrm{~J} 25$

\section{Contents}

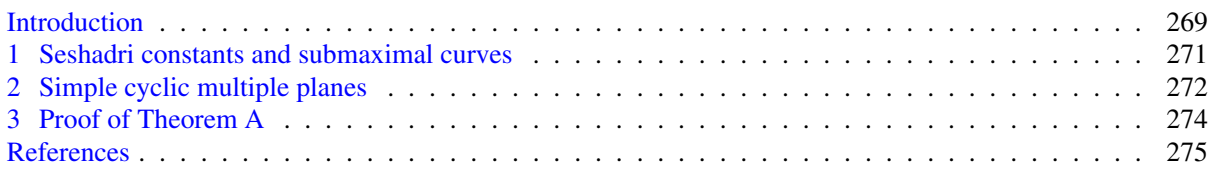

\section{Introduction}

Seshadri constants measure local positivity of line bundles. In this capacity they have close ties with two major conjectures in the theory of smooth complex surfaces: the Segre-Harbourne-

Dedicated to Fabrizio Catanese on the occasion of his 70th birthday.

Sönke Rollenske

rollenske@mathematik.uni-marburg.de

Alex Küronya

kuronya@math.uni-frankfurt.de

1 Institut für Mathematik, Goethe Universität Frankfurt, Robert-Mayer-Str. 6-10., 60325 Frankfurt am Main, Germany

2 FB 12/Mathematik und Informatik, Philipps-Universität Marburg, Hans-Meerwein-Str. 6, 35032 Marburg, Germany 
Gimigliano-Hirschowitz conjecture [11,14-16,23] and the Bounded Negativity Conjecture $[3,4]$.

A big line bundle is locally positive at a point, if the global sections of a suitable multiple of it embed an open neighbourhood of the point into projective space [6,17]. If this is the case then one can try to measure how many 'reasonably different' global sections there are around the reference point.

One way to quantify local positivity is via Seshadri constants defined for ample line bundles by Demailly [10] during his first attempt at Fujita's global generation conjecture. For a thorough introduction and references we refer the reader to [2] and [19, Chapter 5].

Rationality of Seshadri constants is a long-standing open question, and although it is widely assumed to be false, there is no counterexample even in higher dimensions. As examplified by the volume or asymptotic cohomology of a line bundle [5] or Newton-Okounkov bodies [18], asymptotic invariants of linear series have a tendency to be rational on surfaces and wildly irrational in higher dimensions. The existing evidence in the case of Seshadri constants is very mixed.

On the one hand all computed cases of Seshadri constants are rational, and this includes all abelian surfaces, some of which have round nef cones. At the same time there is no structural result pointing towards rationality, although the rationality of Seshadri constants would disprove Nagata's conjecture [11], which is more or less unanimously expected to be true.

Seshadri constants on general surfaces of degree $d \geq 5$ in projective space are expected to be integral multiples of $\sqrt{d}$, hence often irrational. There is a closely related work centering around Szemberg's conjecture [13,24] on primitive solutions to Pell's equation and optimal lower bounds on Seshadri constants.

Here we initiate a study of a class of surfaces that avoided being studied from this point of view.

Theorem A Fix integers $d \geq 2$ and $m \geq 3$. Let $X$ be a smooth hypersurface of degree $m d$ in $\mathbb{P}(1,1,1, m)$ not passing through the singular point. Let $L=\mathcal{O}_{X}(1)$, and $x$ a point on $X$. If the pair $(X, x)$ is general then

$$
\sqrt{d}-\frac{d}{m} \leq \varepsilon(L ; x) \leq \sqrt{d}=\sqrt{L^{2}} .
$$

In particular, for every integer $d$ and $\delta>0$ there exists a point $x$ on a polarised surface $(X, L)$ such that $L^{2}=d$ and $\varepsilon(L ; x) \in\left[\sqrt{L^{2}}-\delta, \sqrt{L^{2}}\right]$.

This result grew out of an attempt to decide the rationality question for this class of surfaces but in the end we had to settle for the above approximation, which leaves the possibility open that $\varepsilon(L, x)=\sqrt{d}$ for the general such surface.

The main step is to prove the estimate in the special case when $B$ is a very general plane curve of degree $m d, X=X_{d, m} \rightarrow \mathbb{P}^{2}$ the simple cyclic $d$-uple cover branched over $B$ and $L=\pi^{*} \mathcal{O}_{\mathbb{P}^{2}}(1)$. One can then deduce (*) directly for a very general point of $X$ using a result of Bauer [2] and the following information on the Picard group.

Theorem B A very general simple cyclic cover X of the plane is a smooth surface with Picard number $\rho(X)=1$.

This was proved by Cox for the full family of hypersurfaces of degree $m d$ in $\mathbb{P}(1,1,1, m)$ in [9] and we show in Proposition 2.2 that his method restricts well to the subfamily we are interested in. 
We work over the complex number field. A variety is an integral separated scheme of finite type over $\mathbb{C}$. A polarized variety $(X, L)$ is a projective variety $X$ equipped with an ample line bundle $L$.

\section{Seshadri constants and submaximal curves}

Here we collect the necessary material about Seshadri constants on surfaces. Good references for this topic are [2] and [19, Chapter 5]. Let $(X, L)$ be a polarised algebraic surface and $C \subset X$ be a curve containing a point $x$. We then define

$$
\varepsilon_{C, x}:=\frac{C \cdot L}{\operatorname{mult}_{x}(C)}
$$

and call $C$ a submaximal curve for $L$ at $x$ if $\varepsilon_{C, x}<\sqrt{L^{2}}$. The Seshadri constant $\varepsilon(X, L ; x)$ (often just $\varepsilon(L ; x)$ ) of $L$ at the point $x \in X$ is defined as

$$
\varepsilon(X, L ; x) \stackrel{\text { def }}{=} \inf _{x \in C} \varepsilon_{C, x} .
$$

Equivalently, upon writing $\pi: \widetilde{X} \rightarrow X$ for the blowing-up of $X$ at $x$ with exceptional divisor $E$, one has

$$
\varepsilon(L ; x)=\sup \left\{t>0 \mid \pi^{*} L-t E \text { is nef }\right\} .
$$

The definition gives rise to the following quick upper bound:

$$
\varepsilon(L ; x) \leq \sqrt{\left(L^{2}\right)} .
$$

It is known that if the inequality is sharp then there must exist a submaximal curve $C \subseteq X$. Equivalently, submaximal means that the proper transform $\widetilde{C}$ of $C$ on $\widetilde{X}$ satisfies $\left(\left(\pi^{*} L-\right.\right.$ $\varepsilon(L ; x) E) \cdot \widetilde{C})=0$.

A couple of words about the behaviour of $\varepsilon(L ; x)$ in its two arguments. As can be seen from its definition as nef threshold on the blow-up, $\varepsilon(L ; x)$ only depends on the numerical equivalence class of $L$, and

$$
\varepsilon(m L ; x)=m \cdot \varepsilon(L ; x)
$$

for all positive integers $m$. In particular, if $\rho(X)=1$, then $\varepsilon(L ; x)$ for the ample generator $L$ of $X$ determines all Seshadri constants at $x \in X$.

By [21] Seshadri constant are upper-semicontinuous in $x$. Consequently, for given $L$, the numbers $\varepsilon(L ; x)$ are constant for very general $x \in X$. It is customary to write $\varepsilon(L ; 1)$ for this common value. If $\rho(X)=1$ then we will denote by $\varepsilon(X, 1)$ the Seshadri constant of the ample generator of $X$ at a very general point of $X$. In this case we will call $\varepsilon(X ; 1)$ the Seshadri constant of the surface $X$.

Usually one is especially interested in irreducible submaximal curves but it is sometimes convenient to allow $C$ to be any curve containing $x$.

Let $H$ be the class of a line in the plane. The proof of the following is an elementary computation using the projection formula.

Lemma 1.1 Let $\pi: X \rightarrow \mathbb{P}^{2}$ be ad-uple plane (that is, a finite surjective morphism of degree d), with polarisation $L=\pi^{*} H$. If $C$ is a plane curve then $\pi^{*} C$ is not submaximal for any point on $X$. 
A key ingredient in our argument is the following result of Thomas Bauer, that controls the degree of a submaximal curve.

Theorem 1.2 (Bauer, [2, Thm. 4.1]) Let $(X, L)$ be a polarised surface and $x \in X$ a very general point. If $C$ is an irreducible submaximal curve for $x$, then

$$
\text { L.C }<\frac{L^{2}}{\sqrt{L^{2}}-\varepsilon_{C, x}} .
$$

\section{Simple cyclic multiple planes}

A $d$-uple plane is a finite map $\pi: X \rightarrow \mathbb{P}^{2}$ of degree $d \geq 2$. In general, the structure of such maps is quite complicated if $d>2$ but we only discuss the following simple construction, where $X$ arises by taking the $d$-th root of a section of a line bundle. More precisely, let $m \geq 1$ and $B$ be a plane curve of degree $m d$ defined by an equation $f$. Then a simple cyclic cover $X$ is a hypersurface of degree $m d$ in $\mathbb{P}(1,1,1, m)$, with equation

$$
w^{d}+f(x, y, z)=0 .
$$

Note that $\pi: X \rightarrow \mathbb{P}^{2}$ is a Galois ramified cover with group $\mathbb{Z} / d \mathbb{Z}$. Decomposing $\pi_{*} \mathcal{O}_{X}$ into eigensheaves with respect to this action we get a decomposition

$$
\pi_{*} \mathcal{O}_{X}=\bigoplus_{k=0}^{d-1} \mathcal{O}_{\mathbb{P}^{2}}(-k m)=\left(\bigoplus_{k \geq 0} \mathcal{O}_{\mathbb{P}^{2}}(-k m) w^{k}\right) /\left(w^{d}-f\right),
$$

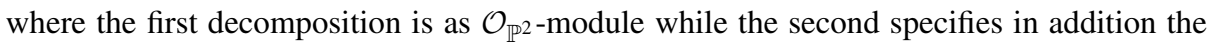
algebra structure. A local computation shows that $X$ is smooth if and only if $B$ is smooth.

For a more complex analytic point view on simple cyclic covers see [1, Ch. 1], for a more general theory of abelian covers compare [22].

Definition 2.1 A simple cyclic $d$-uple plane is a ramified cover

$$
\pi: X=X_{d, m} \rightarrow \mathbb{P}^{2}
$$

of degree $d$, branched over over a curve $B$ of degree $d m$ arising as above. We say $X$ is very general if $B$ is very general.

For lack of convenient reference we sketch a proof of the following.

Proposition 2.2 (Theorem B) Let $d \geq 2$ and $m \geq 3$ be integers. Then a very general simple cyclic d-uple plane $\pi: X \rightarrow \mathbb{P}^{2}$ with branch curve $B$ of degree dm has Picard rank 1 .

This is proved for the full family of weighted hypersurfaces in [9] and we follow the proof very closely. The case of degree 2 is covered in [7]. We will consider $d$-uple planes $X_{d, m}$ as hyperplanes in $\mathbb{P}(1,1,1, m)$ with equation as in (1.2). As explained in the first paragraph of [9, Proof of Theorem] (see also [8, III (a)]), in order to verify that $\rho\left(X_{d, m}\right)=1$ for the general hypersurface, it suffices to check that the map

$$
H^{1}\left(X, \Theta_{X}\right)_{0} \otimes H^{2,0}(X) \longrightarrow H^{1,1}(X)
$$

is surjective, where $H^{1}\left(X, \Theta_{X}\right)_{0}$ stands for the image of $H^{1}\left(X, \Theta_{X}\right)$ under the KodairaSpencer map of the family of degree $d$ hypersurfaces in $\mathbb{P}(1,1,1, m)$ with Eq. (1.2). 
As far as the link between various cohomology spaces occurring above and the graded polynomial ring $S \stackrel{\text { def }}{=} \mathbb{C}[x, y, z, w]$ goes, write

$$
R \stackrel{\text { def }}{=} S /(\nabla F)=S /\left(w^{d-1}, \nabla f\right),
$$

then

$$
\begin{aligned}
H^{2,0}(X) & \simeq R^{d m-(1+1+1+m)}=R^{d m-m-3}, \\
H^{1,1}(X) & \simeq R^{2 d m-m-3}, \text { and } \\
H^{1}\left(X, \Theta_{X}\right)_{0} & \simeq R^{d m} .
\end{aligned}
$$

For proofs see the reference in [9].

Proof Consider the weighted polynomial rings $S^{\prime}=\mathbb{C}[x, y, z] \subset S=\mathbb{C}[x, y, z, w]$, where $x, y, z$ have degree 1 and $w$ has degree $m$. Let $f$ be the equation defining $B$ so that $X$ is cut out by $F=w^{d}+f \in S=\mathbb{C}[x, y, z, w]$. Consider the Milnor algbra

$$
R=S /(\nabla F)=S /\left(w^{d-1}, \nabla f\right),
$$

which is the homomorphic image of $T=S /\left(w^{d-1}\right)$. Let $V$ be the tangent space to the family of $d$-uple planes at $X$. Then as in [9] the result follows if the composition

$$
V \otimes H^{2,0}(X) \stackrel{\kappa \otimes \text { id }}{\longrightarrow} H^{1}\left(\Theta_{X}\right) \otimes H^{2,0}(X) \longrightarrow H^{1,1}(X)
$$

is surjective, where $\kappa$ is the Kodaira-Spencer map. Considering $d$-uple planes as particular hypersurfaces and argueing as in [9], this multiplication map fits into a commutative diagram

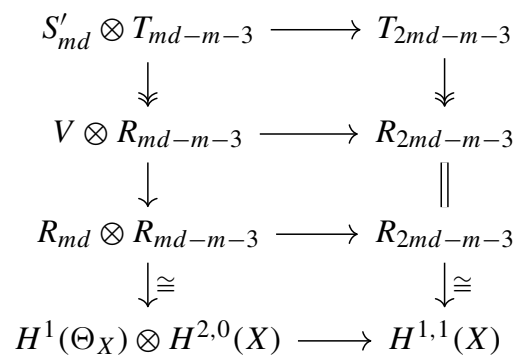

Now note that as a graded $S^{\prime}$-module $T_{k}=\bigoplus_{i=0}^{d-2} w^{i} \cdot S_{k-i m}^{\prime}$ and all summands on the right hand side are non-zero as soon as $k \geq m(d-2)=m(d-1)-m$. Since the multiplication maps in the standard polynomial ring $S^{\prime}$ are surjective, the surjectivity of the first row of the diagram, and hence our claim, follows if $m \geq 3$.

We would like to have that the Picard group is actually generated by $L$, at least for large $m$, but we do not know a convenient reference for this. For our purpose the following simple observation is enough.

Corollary 2.3 Let $\pi: X \rightarrow \mathbb{P}^{2}$ be a very general simple cyclic $d$-uple plane and $L=$ $\pi^{*} \mathcal{O}_{\mathbb{P}^{2}}(1)$. If $C$ is any curve on $X$ then there exists a $k$ such that $d C \in|k L|$.

Proof Let $\sigma$ be a generator of the Galois group of $\pi$. Since the Picard rank is 1 , the automorphisms act trivially on the Picard group and we have

$$
d C \sim \sum_{k=1}^{d}\left(\sigma^{k}\right)^{*} C=\pi^{*} \pi_{*} C \in\left|\pi^{*} \mathcal{O}_{\mathbb{P}^{2}}(k)\right|=|k L|
$$

for some $k$. 


\section{Proof of Theorem A}

We start by proving the estimate $\left(*\right.$ ) for a very general simple cyclic $d$-uple plane $\pi: X \rightarrow \mathbb{P}^{2}$ branched over a curve of degree $m d$.

Assume $C$ is an irreducible curve which is submaximal for a very general point $x \in X$ and such that $\sqrt{L^{2}}-\varepsilon_{C, x}>\frac{d}{m}$. By Corollary 2.3 we have $d C \in|k L|$ and $d C$ is submaximal as well. Then by Bauer's bound, Theorem 1.2, we have

$$
k=\frac{1}{d} k L^{2}=L . C<\frac{d}{\sqrt{d}-\varepsilon_{C, x}}<m .
$$

This inequality implies by the projection formula

$$
H^{0}(X, k L) \cong \bigoplus_{i=0}^{d-1} H^{0}\left(\mathbb{P}^{2},(k-i m) H\right)=H^{0}\left(\mathbb{P}^{2}, k H\right),
$$

that is, every curve of degree at most $m$ is pullback of a plane curve. By Lemma 1.1 the curve $d C$ is not submaximal-a contradiction.

Now consider the family $\mathcal{X} \subset\left|\mathcal{O}_{\mathbb{P}(1,1,1, m)}(m d)\right|$ of smooth hypersurfaces of degree $m d$ in $\mathbb{P}(1,1,1, m)$ not containing the singular point and let

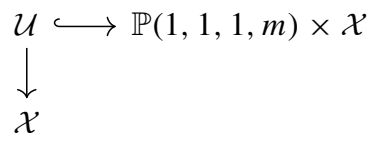

be the universal family. Let $\tilde{\mathcal{U}} \rightarrow \mathcal{U} \times \mathcal{X} \mathcal{U}$ be the blow-up of the diagonal and $E$ the exceptional divisor of the blow up. Then

$$
f: \tilde{\mathcal{U}} \rightarrow \mathcal{U}
$$

is a smooth family of surfaces. If we consider a point in $\mathcal{U}$ as a pair $(X, x)$ of a hypersurface $X$ and a point $x \in X$, then $\tilde{X}=f^{-1}(X, x)$ is exactly the surface $X$ blown up at the point $x$, with exceptional divisor $E_{x}=E \cap \tilde{X}$. Let $\mathcal{L}$ be the pullback of $\mathcal{O}_{\mathbb{P}(1,1,1, m)}(1)$ to $\mathcal{U}$; it is a line bundle on every fibre by our assumptions.

Now note that the simple cyclic $d$-uple planes branched over a curve of degree $m d$ are contained in this family and in particular, there is a point $(X, x) \in \mathcal{U}$ such that $\varepsilon\left(\left.\mathcal{L}\right|_{X} ; x\right) \geq$ $\sqrt{d}-\frac{d}{m}$. By the characterisation of Seshadri constants via nefness on the blow up in (1.1) we have that

$$
\left.\left(f^{*} \mathcal{L}-\varepsilon\left(\left.\mathcal{L}\right|_{X} ; x\right) E\right)\right|_{f^{-1}(X, x)}
$$

is nef. By [20, Corollary 4] there is an open subset $\mathcal{U}^{\prime} \subset \mathcal{U}$ containing $(X, x)$ and such that for every $(Y, y) \in \mathcal{U}^{\prime}$ the line bundle $\left.\mathcal{L}\right|_{Y}-\varepsilon\left(\left.\mathcal{L}\right|_{X} ; x\right) E_{y}$ is nef.

Using (1.1) again, we see that $\varepsilon\left(\left.\mathcal{L}\right|_{X} ; x\right) \leq \varepsilon\left(\left.\mathcal{L}\right|_{Y} ; y\right)$. Therfore the estimate $(*)$ holds for all pairs in the open subset $\mathcal{U}^{\prime}$. This concludes the proof of Theorem $\mathrm{A}$.

Acknowledgements We are grateful to Thomas Bauer and Jakob Stix for helpful discussions. This cooperation started during the Workshop on local negativity and positivity on algebraic surfaces in 2017 in Hannover, we thank the organizers Roberto Laface and Piotr Pokora for the invitation. The first-named author was partially supported by the LOEWE grant 'Uniformized structures in Arithmetic and Geometry'.

Funding Open Access funding enabled and organized by Projekt DEAL. 
Open Access This article is licensed under a Creative Commons Attribution 4.0 International License, which permits use, sharing, adaptation, distribution and reproduction in any medium or format, as long as you give appropriate credit to the original author(s) and the source, provide a link to the Creative Commons licence, and indicate if changes were made. The images or other third party material in this article are included in the article's Creative Commons licence, unless indicated otherwise in a credit line to the material. If material is not included in the article's Creative Commons licence and your intended use is not permitted by statutory regulation or exceeds the permitted use, you will need to obtain permission directly from the copyright holder. To view a copy of this licence, visit http://creativecommons.org/licenses/by/4.0/.

\section{References}

1. Barth, W.P., Hulek, K., Peters, C.A.M., de Xen, A.: Compact Complex Surfaces. 2nd Enlarged ed. Ergebnisse der Mathematik und ihrer Grenzgebiete. 3. Folge 4, p. 436. Springer, Berlin (2004)

2. Bauer, T.: Seshadri constants on algebraic surfaces. Math. Ann. 313(3), 547-583 (1999)

3. Bauer, Th., Bocci, C., Cooper, S., Di Rocco, S., Harbourne, B., Jabbusch, K., Küronya, A., Knutsen, A.L., Miranda, R., Schenk, H., Szemberg, T., Teitler, Z.: Recent developments and open problems in the theory of linear series. In: Piotr, P. (ed) Contributions to Algebraic Geometry, IMPANGA Lecture Notes, EMS Series of Congress Reports, edited by the European Mathematical Society Publishing House, pp 93-140 (2012)

4. Bauer, T., Harbourne, B., Knutsen, A.L., Küronya, A., Müller-Stach, S., Roulleau, X., Szemberg, T.: Negative curves on algebraic surfaces. Duke Math. J. 162(10), 1877-1894 (2013)

5. Bauer, T., Küronya, A., Szemberg, T.: Zariski decompositions, volumes, and stable base loci. J. Reine Angew. Math. 576, 209-233 (2004)

6. Boucksom, S., Cacciola, S., Lopez, A.F.: Augmented base loci and restricted volume on normal varieties. Math. Z. 278(3-4), 979-985 (2014)

7. Buium, A.: Sur le nombre de Picard des revêtements doubles des surfaces algébriques. C. R. Acad. Sci. Paris Sér. I Math. 296(8), 361-364 (1983)

8. Carlsson, J., Green, M., Griffiths, P., Harris, J.: Infinitesimal variation of Hodge structure I. Comp. Math. 50, 109-205 (1983)

9. Cox, D.A.: Picard numbers of surfaces in 3-dimensional weighted projective spaces. Math. Z. 201(2), 183-189 (1989)

10. Demailly, J.-P.: Singular Hermitian metrics on positive line bundles, complex algebraic varieties (Bayreuth, 1990). Springer LNM 1507, 87-104 (1992)

11. Dumnicki, M., Küronya, A., Maclean, C., Szemberg, T.: Rationality of Seshadri constants and the SegreHarbourne-Gimigliano-Hirschowitz conjecture. Adv. Math. 303, 1162-1170 (2016)

12. Ein, L., Lazarsfeld, R., Mustaţă, M., Nakamaye, M., Popa, M.: Asymptotic base loci of linear series. Ann. Inst. Fourier (Grenoble) 56(6), 1701-1734 (2006)

13. Farnik, Ł., Szemberg, T., Szpond, J., Tutaj-Gasińska, H.: Restrictions on Seshadri constants on surfaces. Taiwan. J. Math. 21(1), 27-41 (2017)

14. Gimigliano, A.: On linear systems of plane curves, Thesis, Queen's University, Kingston (1987)

15. Harbourne, B.: The geometry of rational surfaces and Hilbert functions of points in the plane. In: Proceedings of the 1984 Vancouver Conference in Algebraic Geometry, CMS Conf. Proc. 6, American Mathematical Society, Providence, RI, pp 95-111 (1986)

16. Hirschowitz, A.: Une conjecture pour la cohomologie des diviseurs sur les surfaces rationelles génériques. J. Reine Angew. Math. 397, 208-213 (1989)

17. Küronya, A., Lozovanu, V.: Geometric aspects of Newton-Okounkov bodies. In: Buczynski, J., Cynk, S., Szemberg, T. (eds.) Phenomenological Approach to Algebraic Geometry, Banach Center Publications, vol. 116, pp. 137-212. Warsaw, Polish Academy of Sciences (2018)

18. Küronya, A., Lozovanu, V., Maclean, C.: Convex bodies appearing as Okounkov bodies of divisors. Adv. Math. 229, 2622-2639 (2012)

19. Lazarsfeld, R.: Positivity in Algebraic Geometry. I., Ergebnisse der Mathematik und ihre Grenzgebiete. 3. Folge, vol. 48. Springer, Berlin (2004)

20. Moriwaki, A.: A criterion of openness of a family of NEF line bundles. Manuscr. Math. 75(3), 327-331 (1992)

21. Oguiso, K.: Seshadri constants in a family of surfaces. Math. Ann. 323(4), 625-631 (2002)

22. Pardini, R.: Abelian covers of algebraic varieties. J. Reine Angew. Math. 417, 191-213 (1991)

23. Segre, B.: Alcune questioni su insiemi finiti di punti in geometria algebrica. Atti Convegno Int. Geometria Algebrica ((Torino, : Rattero. Turin) 1962, 15-33 (1961) 
24. Szemberg, T.: Bounds on Seshadri constants on surfaces with Picard number 1. Commun. Algebra 40(7), 2477-2484 (2012)

Publisher's Note Springer Nature remains neutral with regard to jurisdictional claims in published maps and institutional affiliations. 